\title{
Efficacy of Tiotropium + Olodaterol in Patients with Chronic Obstructive Pulmonary Disease by Initial Disease Severity and Treatment Intensity: A Post Hoc Analysis
}

Gary T. Ferguson · Matjaž Fležar · Stephanie Korn · Lawrence Korducki •

Lars Grönke · Roger Abrahams · Roland Buhl

To view enhanced content go to www.advancesintherapy.com

Received: April 24, 2015 / Published online: June 26, 2015

(C) The Author(s) 2015. This article is published with open access at Springerlink.com

\section{ABSTRACT}

Introduction: The once-daily long-acting muscarinic antagonist (LAMA) tiotropium and once-daily long-acting $\beta_{2}$-agonist (LABA) olodaterol have been studied as a once-daily fixed-dose combination (FDC) in patients with chronic obstructive pulmonary disease (COPD).

Electronic supplementary material The online version of this article (doi:10.1007/s12325-015-0218-0) contains supplementary material, which is available to authorized users.

G. T. Ferguson $(\bowtie)$

Pulmonary Research Institute of Southeast

Michigan, Livonia, MI, USA

e-mail: garytferguson@msn.com

M. Fležar

Klinika Golnik, Golnik, Slovenia

S. Korn · R. Buhl

Pulmonary Department, Mainz University Hospital,

Mainz, Germany

L. Korducki

Boehringer Ingelheim Pharmaceuticals Inc.,

Ridgefield, CT, USA

L. Grönke

Boehringer Ingelheim Pharma GmbH and Co. KG, Ingelheim am Rhein, Germany

R. Abrahams

Morgantown Pulmonary Associates, Morgantown, WV, USA
Two large, 52-week, double-blind, parallelgroup studies in patients with moderate-very severe COPD demonstrated that tiotropium + olodaterol significantly improved lung function and symptoms versus the monocomponents. This post hoc analysis determined effects on lung function by prior LAMA or LABA maintenance treatment and initial disease severity.

Methods: 5162 patients were randomized and treated with olodaterol $5 \mu \mathrm{g}$, tiotropium $2.5 \mu \mathrm{g}$, tiotropium $5 \mu \mathrm{g}$, tiotropium + olodaterol 2.5/ $5 \mu \mathrm{g}$, or tiotropium + olodaterol $5 / 5 \mu \mathrm{g}$ (all once daily via Respimat ${ }^{\circledR}$ inhaler). Primary efficacy (lung-function) end points were forced expiratory volume in $1 \mathrm{~s}\left(\mathrm{FEV}_{1}\right)$ area under the curve from 0 to $3 \mathrm{~h}\left(\mathrm{AUC}_{0-3}\right)$ and trough $\mathrm{FEV}_{1}$ responses (i.e., change from baseline). Pooled data are presented for the following subgroups: prior maintenance treatment with LAMA or LABA, Global initiative for chronic Obstructive Lung Disease (GOLD) 2 (predicted $\mathrm{FEV}_{1} 50 \%$ to $<80 \%)$ and 3 (30\% to $<50 \%) / 4(<30 \%)$, sex, age, and prior use of inhaled corticosteroids.

Results: Tiotropium + olodaterol FDC improved lung function over the monocomponents in patients with GOLD 2 
and 3-4 disease, irrespective of prior LAMA or LABA maintenance therapy; most comparisons between FDCs and their respective monocomponents were statistically significant $(P<0.05) . \quad \mathrm{FEV}_{1} \quad \mathrm{AUC}_{0-3}$ and trough $\mathrm{FEV}_{1}$ responses for the individual treatments were generally greater in patients with less severe COPD at baseline.

Conclusions: Tiotropium + olodaterol $\quad 5 / 5 \mu \mathrm{g}$ significantly improved $\mathrm{FEV}_{1} \quad \mathrm{AUC}_{0-3}$ and trough $\mathrm{FEV}_{1}$ in all GOLD severity groups compared to olodaterol $5 \mu \mathrm{g}$ and tiotropium $5 \mu \mathrm{g}$ alone, irrespective of whether patients had received prior LAMA or LABA maintenance treatment. Improvements from baseline in lung function were generally greater in patients with less severe disease.

Funding: Boehringer Ingelheim.

Trial registration: ClinicalTrials.gov numbers, NCT01431274 and NCT01431287.

Keywords: Bronchodilator; COPD; Disease severity; Lung function; Subgroup; Tiotropium + olodaterol

\section{INTRODUCTION}

Bronchodilators, including long-acting muscarinic antagonists (LAMAs) and longacting $\beta_{2}$-agonists (LABAs), are central to the maintenance treatment of patients with moderate to very severe chronic obstructive pulmonary disease (COPD) [1]. The once-daily LAMA tiotropium is well established in the treatment of COPD and improves the main functional and patient-orientated outcomes of the disease [2-7]. Olodaterol is a novel oncedaily LABA that provides 24 -h bronchodilation [8-11], symptomatic benefit [12], and enhanced exercise capacity [13]. Tiotropium + olodaterol has recently been evaluated in a phase III program to determine its efficacy in the treatment of COPD [14, 15].

International guidelines indicate that combining bronchodilators of different pharmacologic classes may improve efficacy and reduce the risk of side effects, rather than increasing the dose of a single bronchodilator. The introduction of combination products raises questions regarding the timing of their initiation during the course of COPD, with recent data indicating the potential benefits of earlier pharmacologic intervention with combination bronchodilator medications [1620].

The pivotal phase III trials TONADO- 1 and -2 have demonstrated that once-daily tiotropium + olodaterol significantly improved lung function and health-related quality of life compared to the monocomponents over 1 year in patients with moderate to very severe COPD [14]. These studies were notable in that they included a higher proportion of patients with more severe disease (Global initiative for chronic Obstructive Lung Disease [GOLD] 3 and 4) compared to previously reported studies of other LAMA and LABA combinations such as indacaterol + glycopyrronium $[21,22]$, and thus studied a broader population with respect to disease severity.

While significant responses were observed in the overall population of TONADO, evaluating the effectiveness of tiotropium + olodaterol in specific subgroups of patients is of clear interest to help inform health care providers. This is particularly pertinent in light of the fact that in clinical practice the population of patients with COPD is heterogeneous with respect to factors such as severity of disease, age, and concurrent COPD medication usage. The large nature of the TONADO studies offers the opportunity to study such subgroups. This post hoc analysis of the efficacy data from TONADO- 1 and -2 was 
conducted to help understand these points, considering the effectiveness of tiotropium + olodaterol across COPD disease severities, in patients with or without prior bronchodilator or inhaled corticosteroid (ICS) use, and according to patients' age and sex. As this analysis is post hoc, all $P$ values and confidence intervals are nominal.

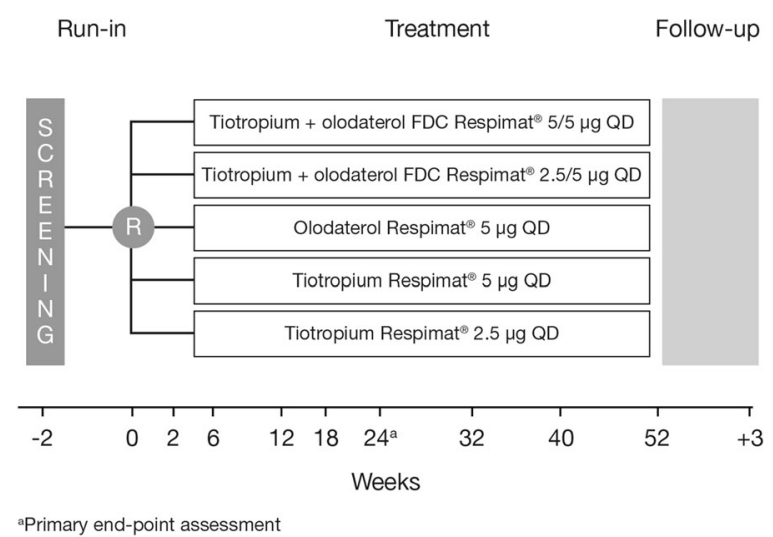

Fig. 1 Study design (Study 1237.5: NCT01431274; Study 1237.6: NCT01431287). $R$ randomization, FDC fixeddose combination, $Q D$ once daily

\section{METHODS}

\section{Study Design}

Two replicate, multinational, phase III, randomized, double-blind, active-controlled trials were conducted to evaluate the efficacy and safety of tiotropium + olodaterol in comparison to the monotherapy components (TONADO-1, Study 1237.5: NCT01431274; TONADO-2, Study 1237.6: NCT01431287) (Fig. 1).

After a screening visit and 2-week baseline period, patients were randomized to receive tiotropium + olodaterol $(2.5 / 5$ or $5 / 5 \mu \mathrm{g})$ or the individual components as monotherapy (tiotropium 2.5 or $5 \mu \mathrm{g}$, or olodaterol $5 \mu \mathrm{g}$ ) via the Respimat ${ }^{\circledR}$ Soft Mist ${ }^{\mathrm{TM}}$ inhaler (Boehringer Ingelheim, Ingelheim am Rhein, Germany) for 52 weeks. Due to the length of therapy and the severity of disease studied, placebo was not used as a treatment arm in these studies. Patients

\section{Key inclusion criteria \\ Key exclusion criteria \\ Male or female outpatients with a history of moderate to very severe COPD (GOLD 2-4) \\ Aged $\geq 40$ years \\ Current or ex-smokers with a smoking history of $>10$ pack-years \\ Post-bronchodilator $\mathrm{FEV}_{1}<80 \%$ of predicted normal Post-bronchodilator $\mathrm{FEV}_{1} /$ forced vital capacity $<70 \%$ \\ History of asthma \\ Significant disease other than COPD \\ Myocardial infarction within 1 year of screening Unstable or life-threatening cardiac arrhythmia Known active tuberculosis; clinically evident bronchiectasis; cystic fibrosis or life-threatening pulmonary obstruction \\ Hospitalization for heart failure within the past year; diagnosed thyrotoxicosis or paroxysmal tachycardia \\ Previous thoracotomy with pulmonary resection \\ Regular use of daytime oxygen if patients were unable to abstain during clinic visits \\ Current enrollment in a pulmonary rehabilitation program (or completed in prior 6 weeks)}

Fig. 2 Key inclusion and exclusion criteria. COPD chronic obstructive pulmonary disease, GOLD Global initiative for chronic Obstructive Lung Disease, $F E V_{1}$ forced expiratory volume in $1 \mathrm{~s}$ 
receiving ICS at the start of the study could continue with their medication and all patients were provided with salbutamol/albuterol metered-dose inhaler (100 $\mu$ g per actuation) as rescue medication, as required during the trial. Patients receiving a LAMA or LABA prior to the study were required to discontinue these medications during screening. The studies

Table 1 Demographic and baseline patient characteristics (treated population): combined data for patients without prior LAMA or LABA treatment at baseline, stratified by initial GOLD stage ${ }^{a}$

\begin{tabular}{|c|c|c|c|c|c|}
\hline & $\begin{array}{l}\text { Olodaterol } \\
5 \mu \mathrm{g}\end{array}$ & $\begin{array}{l}\text { Tiotropium } \\
2.5 \mu \mathrm{g}\end{array}$ & $\begin{array}{l}\text { Tiotropium } \\
5 \mu \mathrm{g}\end{array}$ & $\begin{array}{l}\text { Tiotropium }+ \\
\text { olodaterol 2.5/ } \\
5 \mu \mathrm{g}\end{array}$ & $\begin{array}{l}\text { Tiotropium }+ \\
\text { olodaterol } \\
5 / 5 \mu \mathrm{g}\end{array}$ \\
\hline \multicolumn{6}{|l|}{ GOLD $2(n=1148)$} \\
\hline Participants, $n$ & 238 & 231 & 240 & 211 & 228 \\
\hline Male, $n(\%)$ & $171(71.8)$ & $161(69.7)$ & $160(66.7)$ & $156(73.9)$ & $148(64.9)$ \\
\hline Mean (SD) age, years & $63.6(8.5)$ & $63.2(8.9)$ & $62.5(8.9)$ & $64.4(8.2)$ & $63.0(8.2)$ \\
\hline Current smoker, $n$ (\%) & $107(45.0)$ & $116(50.2)$ & $112(46.7)$ & $88(41.7)$ & $111(48.7)$ \\
\hline \multicolumn{6}{|l|}{ Post-bronchodilator screening } \\
\hline Mean (SD) $\mathrm{FEV}_{1}, \mathrm{~mL}$ & $1773(483)$ & $1785(478)$ & $1765(474)$ & $1769(441)$ & $1716(435)$ \\
\hline $\begin{array}{l}\text { Mean }(\mathrm{SD}) \text { change from pre- to post- } \\
\text { bronchodilator } \mathrm{FEV}_{1}, \mathrm{~mL}\end{array}$ & $172(174)$ & $210(188)$ & $184(163)$ & $202(160)$ & $175(156)$ \\
\hline Mean (SD) $\mathrm{FEV}_{1} / \mathrm{FVC}, \%$ & $52.6(8.5)$ & $52.7(8.6)$ & $54.5(8.7)$ & $53.0(8.8)$ & $54.2(8.7)$ \\
\hline $\begin{array}{l}\text { Mean (SD) \% of predicted normal } \\
\text { FEV }_{1}\end{array}$ & $63.4(8.2)$ & $63.5(8.1)$ & $63.1(8.4)$ & $63.9(8.3)$ & $63.2(8.8)$ \\
\hline \multicolumn{6}{|l|}{ GOLD 3-4 $(n=973)$} \\
\hline Participants, $n$ & 179 & 199 & 213 & 184 & 198 \\
\hline Male, $n(\%)$ & $144(80.4)$ & $161(80.9)$ & $178(83.6)$ & $142(77.2)$ & $143(72.2)$ \\
\hline Mean $(\mathrm{SD})$ age, years & $63.0(7.8)$ & $62.3(8.1)$ & $62.7(8.7)$ & $63.1(7.4)$ & $62.4(8.4)$ \\
\hline Current smoker, $n$ (\%) & $82(45.8)$ & $78(39.2)$ & $63(29.6)$ & $77(41.8)$ & $74(37.4)$ \\
\hline \multicolumn{6}{|l|}{ Post-bronchodilator screening } \\
\hline Mean (SD) $\mathrm{FEV}_{1}, \mathrm{~mL}$ & $1010(292)$ & $1022(291)$ & $1047(286)$ & $1052(314)$ & $996(284)$ \\
\hline $\begin{array}{l}\text { Mean }(\mathrm{SD}) \text { change from pre- to post- } \\
\text { bronchodilator } \mathrm{FEV}_{1}, \mathrm{~mL}\end{array}$ & $145(116)$ & $140(136)$ & $145(118)$ & $157(111)$ & $139(163)$ \\
\hline Mean (SD) $\mathrm{FEV}_{1} / \mathrm{FVC}, \%$ & $37.9(9.3)$ & $37.3(9.6)$ & $38.3(10.3)$ & $37.3(8.6)$ & $39.2(10.2)$ \\
\hline $\begin{array}{l}\text { Mean (SD) \% of predicted normal } \\
\text { FEV }_{1}\end{array}$ & $36.5(8.8)$ & $36.4(8.5)$ & $36.8(8.5)$ & $37.5(8.8)$ & $36.4(8.6)$ \\
\hline
\end{tabular}

$L A M A$ long-acting muscarinic antagonists, $L A B A$ long-acting $\beta_{2}$-agonist, GOLD Global initiative for chronic Obstructive Lung Disease, $S D$ standard deviation, $F E V_{1}$ forced expiratory volume in $1 \mathrm{~s}, F V C$ forced vital capacity

a Based on post-bronchodilator $\mathrm{FEV}_{1}$ percentage predicted 
included outpatients aged $\geq 40$ years with a history of moderate to very severe COPD (GOLD 2-4). Key inclusion and exclusion criteria are detailed in Fig. 2.

\section{End Points}

The primary spirometry end points were evaluated after 24 weeks of treatment as

Table 2 Demographic and baseline patient characteristics (treated population): combined data for patients with prior LAMA or LABA at baseline, stratified by initial GOLD stage ${ }^{a}$

\begin{tabular}{|c|c|c|c|c|c|}
\hline & $\begin{array}{l}\text { Olodaterol } \\
5 \mu \mathrm{g}\end{array}$ & $\begin{array}{l}\text { Tiotropium } \\
2.5 \mu \mathrm{g}\end{array}$ & $\begin{array}{l}\text { Tiotropium } \\
5 \mu \mathrm{g}\end{array}$ & $\begin{array}{l}\text { Tiotropium }+ \\
\text { olodaterol 2.5/ } \\
5 \mu \mathrm{g}\end{array}$ & $\begin{array}{l}\text { Tiotropium }+ \\
\text { olodaterol } 5 / 5 \\
\mu \mathrm{g}\end{array}$ \\
\hline \multicolumn{6}{|l|}{ GOLD $2(n=1140)$} \\
\hline Participants, $n$ & 294 & 287 & 277 & 308 & 274 \\
\hline Male, $n(\%)$ & $201(68.4)$ & $202(70.4)$ & $180(65.0)$ & $210(68.2)$ & $202(73.7)$ \\
\hline Mean (SD) age, years & $65.4(8.0)$ & $65.3(9.1)$ & $64.8(8.7)$ & $64.7(8.2)$ & $65.3(8.9)$ \\
\hline Current smoker, $n$ (\%) & 87 (29.6) & $102(35.5)$ & $98(35.4)$ & $107(34.7)$ & $98(35.8)$ \\
\hline \multicolumn{6}{|l|}{ Post-bronchodilator screening } \\
\hline Mean (SD) $\mathrm{FEV}_{1}, \mathrm{~mL}$ & $1693(398)$ & $1701(401)$ & $1685(446)$ & $1674(409)$ & $1690(440)$ \\
\hline $\begin{array}{l}\text { Mean (SD) change from pre- to post- } \\
\text { bronchodilator } \mathrm{FEV}_{1}, \mathrm{~mL}\end{array}$ & $203(144)$ & $191(144)$ & $196(170)$ & $195(151)$ & $201(151)$ \\
\hline Mean (SD) $\mathrm{FEV}_{1} / \mathrm{FVC}, \%$ & $52.4(8.5)$ & $52.5(8.4)$ & $51.3(8.7)$ & $50.9(9.2)$ & $51.2(8.7)$ \\
\hline $\begin{array}{l}\text { Mean (SD) \% of predicted normal } \\
\text { FEV }_{1}\end{array}$ & $62.9(8.0)$ & $62.2(8.3)$ & $62.7(8.1)$ & $61.6(7.8)$ & $62.0(7.4)$ \\
\hline \multicolumn{6}{|l|}{ GOLD 3-4 $(n=1597)$} \\
\hline Participants, $n$ & 327 & 313 & 302 & 326 & 329 \\
\hline Male, $n(\%)$ & $248(75.8)$ & $228(72.8)$ & $237(78.5)$ & $249(76.4)$ & $240(72.9)$ \\
\hline Mean (SD) age, years & $64.3(8.2)$ & $64.5(8.2)$ & $64.9(7.8)$ & $64.0(7.3)$ & $64.0(7.7)$ \\
\hline Current smoker, $n$ (\%) & $102(31.2)$ & $92(29.4)$ & $96(31.8)$ & $99(30.4)$ & $117(35.6)$ \\
\hline \multicolumn{6}{|l|}{ Post-bronchodilator screening } \\
\hline Mean (SD) $\mathrm{FEV}_{1}, \mathrm{~mL}$ & $1005(288)$ & $1057(298)$ & $993(277)$ & $1048(295)$ & $1008(277)$ \\
\hline $\begin{array}{l}\text { Mean (SD) change from pre- to post- } \\
\text { bronchodilator } \mathrm{FEV}_{1}, \mathrm{~mL}\end{array}$ & $145(124)$ & $155(122)$ & $154(120)$ & $155(118)$ & $142(120)$ \\
\hline Mean (SD) $\mathrm{FEV}_{1} / \mathrm{FVC}, \%$ & $36.8(8.6)$ & $37.6(8.8)$ & $36.4(8.7)$ & $37.1(8.8)$ & $37.3(8.8)$ \\
\hline $\begin{array}{l}\text { Mean (SD) \% of predicted normal } \\
\text { FEV }_{1}\end{array}$ & $36.8(8.2)$ & $38.3(7.7)$ & $36.2(8.5)$ & $37.8(8.2)$ & $37.0(8.1)$ \\
\hline
\end{tabular}

$L A M A$ long-acting muscarinic antagonists, $L A B A$ long-acting $\beta_{2}$-agonist, GOLD Global initiative for chronic Obstructive Lung Disease, $S D$ standard deviation, $F E V_{1}$ forced expiratory volume in $1 \mathrm{~s}, F V C$ forced vital capacity

a Based on post-bronchodilator $\mathrm{FEV}_{1}$ percentage predicted 
follows: forced expiratory volume in $1 \mathrm{~s}\left(\mathrm{FEV}_{1}\right)$ area under the curve from 0 to $3 \mathrm{~h}\left(\mathrm{AUC}_{0-3}\right)$ and trough $\mathrm{FEV}_{1}$ responses (i.e., change from baseline to 24 weeks). Details of all assessments performed, end points evaluated, statistical methodology, and primary results for these studies have been reported previously [14].

\section{Statistical Methods}

In this analysis, $\mathrm{FEV}_{1} \mathrm{AUC}_{0-3}$ and trough $\mathrm{FEV}_{1}$ were evaluated according to the following subgroups: patients without prior LAMA or LABA treatment at baseline or patients with prior LAMA or LABA treatment at baseline. Within each category of prior LAMA/LABA use, analyses were split according to whether patients had GOLD 2 (predicted $\mathrm{FEV}_{1} 50 \%$ to $<80 \%)$ or GOLD $3(30 \%$ to $<50 \%) / 4(<30 \%)$ COPD at baseline.

Pooled data from TONADO-1 and -2 were used in this analysis. Mean changes from baseline to 24 weeks were analyzed using a mixed-effect model repeated measures approach, including the fixed, categorical effects of treatment, test day, and treatmentby-test-day interaction, as well as the continuous, fixed covariates of baseline and baseline-by-test-day interaction. A spatial power covariance structure was used to model withinpatient errors. The Kenward-Roger approximation was used to estimate denominator degrees of freedom.

In addition, pooled $\mathrm{FEV}_{1} \mathrm{AUC}_{0-3}$ and trough $\mathrm{FEV}_{1}$ responses at 24 weeks were analyzed according to patients' age $(<65,65$ to $<75$, and 75 to $<85$ years), sex, and ICS usage using the model described above. These data are presented as forest plots, generated with SAS Version 9.2 (SAS Institute Inc., Cary, NC, USA).

\section{Compliance with Ethics Guidelines}

This article does not contain any new studies with human or animal subjects performed by any of the authors.
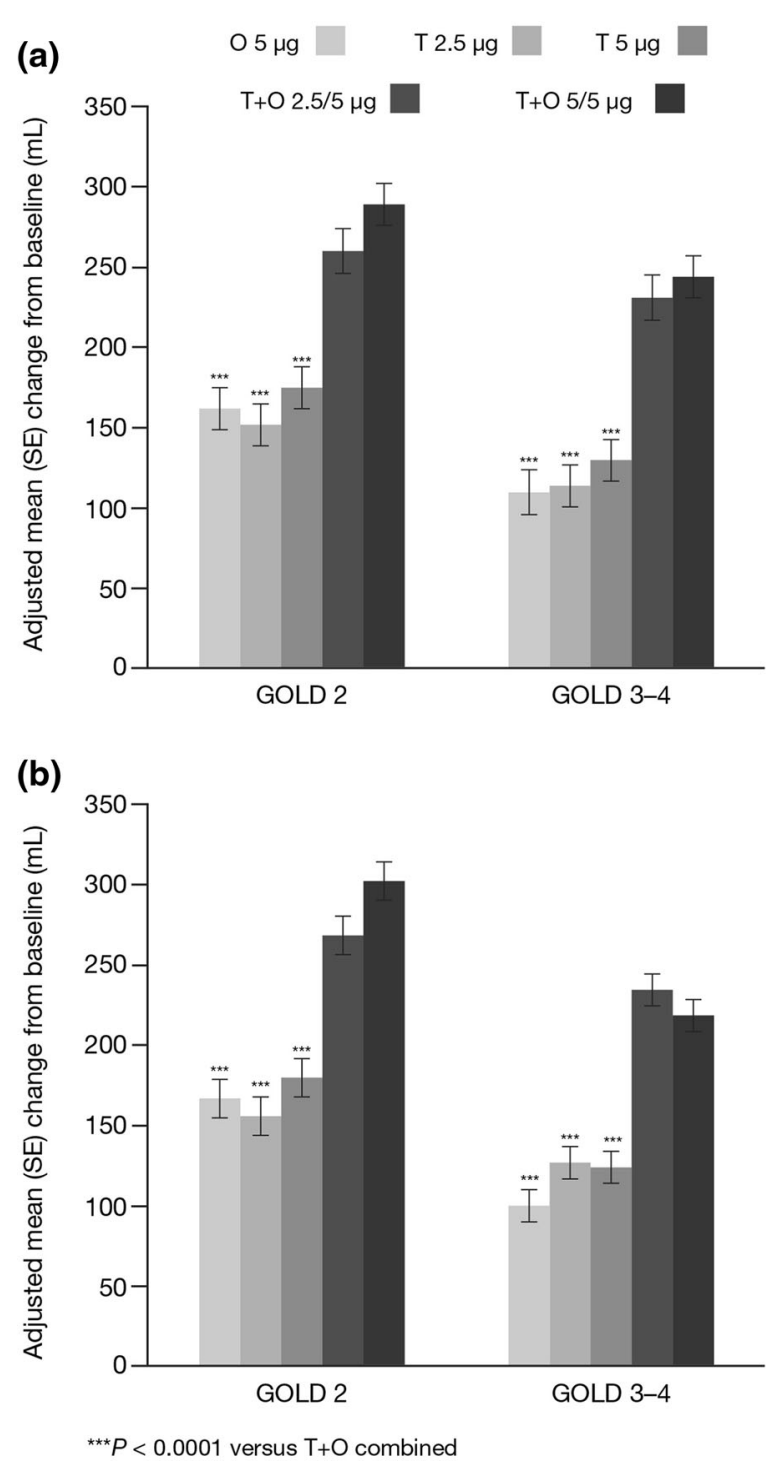

Fig. 3 Adjusted mean $\mathrm{FEV}_{1} \mathrm{AUC}_{0_{-3}}$ responses at 24 weeks; pooled data from TONADO-1 and -2 . a Patients without prior LAMA or LABA use; b patients with prior LAMA or LABA use. $F E V_{1}$ forced expiratory volume in $1 \mathrm{~s}, A U C_{0-3}$ area under the curve from 0 to $3 \mathrm{~h}, L A M A$ long-acting muscarinic antagonist, $L A B A$ long-acting $\beta_{2}$-agonist, $S E$ standard error, $O$ olodaterol, $T$ tiotropium, GOLD Global initiative for chronic Obstructive Lung Disease 


\section{RESULTS}

\section{Patient Disposition and Baseline Characteristics}

A total of 5163 patients were randomized, of whom 5162 received study treatment (2624 Study 1237.5; 2538 Study 1237.6). The baseline characteristics of patients are presented in Tables 1 and 2 based on prior LAMA or LABA maintenance treatment for COPD and GOLD stage.

Overall baseline characteristics were similar across maintenance-treatment subgroups. Lung function in patients with GOLD 2 disease did tend to be slightly better in those who did not

Table 3 Adjusted mean (SE) $\mathrm{FEV}_{1} \mathrm{AUC}_{0_{-3}}$ and trough $\mathrm{FEV}_{1}$ responses (i.e., change from baseline) after 24 weeks of treatment (full analysis set): combined data for patients without prior LAMA or LABA treatment at baseline: treatment differences

\begin{tabular}{lllllll}
\hline $\begin{array}{l}\text { Treatment } \\
\text { comparison }\end{array}$ & $\begin{array}{l}\text { Adjusted mean (SE) } \\
\text { FEV }_{1} \mathrm{AUC}_{0-3}, \mathrm{~mL}\end{array}$ & $95 \% \mathrm{CI}$ & $P$ value & $\begin{array}{l}\text { Adjusted mean }(\mathrm{SE}) \\
\text { trough } \mathrm{FEV}_{1}, \mathrm{~mL}\end{array}$ & $95 \% \mathrm{CI}$ & $P$ value \\
\hline
\end{tabular}

GOLD 2

Tiotropium + olodaterol

$5 / 5 \mu \mathrm{g}$

$\begin{array}{lllllll}\text { versus olodaterol } 5 \mu \mathrm{g} & 127(19) & 90,164 & <0.0001 & 82(20) & 43,120 & <0.0001 \\ \text { versus tiotropium } 5 \mu \mathrm{g} & 114(19) & 77,151 & <0.0001 & 79(20) & 40,118 & <0.0001\end{array}$

Tiotropium + olodaterol

$2.5 / 5 \mu \mathrm{g}$

$\begin{array}{lrrrrrr}\text { versus olodaterol } 5 \mu \mathrm{g} & 98(19) & 61,136 & <0.0001 & 66(20) & 26,105 & 0.0012 \\ \text { versus tiotropium } 2.5 \mu \mathrm{g} & 108(19) & 70,146 & <0.0001 & 71(20) & 31,111 & 0.0005 \\ \text { versus tiotropium } 5 \mu \mathrm{g} & 85(19) & 47,123 & <0.0001 & 63(20) & 23,102 & 0.0020\end{array}$

GOLD 3-4

Tiotropium + olodaterol

$5 / 5 \mu \mathrm{g}$

$\begin{array}{llllllr}\text { versus olodaterol } 5 \mu \mathrm{g} & 134(19) & 96,171 & <0.0001 & 92(20) & 53,131 & <0.0001 \\ \text { versus tiotropium } 5 \mu \mathrm{g} & 114(18) & 79,150 & <0.0001 & 69(19) & 32,106 & 0.0002\end{array}$

Tiotropium + olodaterol

$2.5 / 5 \mu \mathrm{g}$

\begin{tabular}{lllllll} 
versus olodaterol $5 \mu \mathrm{g}$ & $120(19)$ & 82,159 & $<0.0001$ & $53(20)$ & 13,92 & 0.0091 \\
versus tiotropium $2.5 \mu \mathrm{g}$ & $117(19)$ & 80,154 & $<0.0001$ & $44(20)$ & 6,83 & 0.0237 \\
versus tiotropium $5 \mu \mathrm{g}$ & $101(19)$ & 65,137 & $<0.0001$ & $30(19)$ & $-8,67$ & 0.1237 \\
\hline
\end{tabular}

Adjusted mean (SE) obtained from fitting a mixed model for repeated measurements including fixed effects of treatment, planned test day, treatment-by-test-day interaction, baseline, and baseline-by-test-day interaction; patient as a random effect; spatial power covariance structure for within-patient errors and Kenward-Roger approximation of denominator degrees of freedom $S E$ standard error, $F E V_{I}$ forced expiratory volume in $1 \mathrm{~s}, A U C_{0-3}$ area under the curve from 0 to $3 \mathrm{~h}, L A M A$ long-acting muscarinic antagonist, $L A B A$ long-acting $\beta_{2}$-agonist, $C I$ confidence interval, GOLD Global initiative for chronic Obstructive Lung Disease 
receive prior LAMA or LABA maintenance treatment than in those who received maintenance therapies before the study. In addition, more patients who received prior LAMA or LABA maintenance treatment were also taking ICS than those without prior maintenance treatment.

\section{Efficacy}

Patients Without Prior LAMA or LABA

\section{Maintenance Treatment}

In each treatment group, $\mathrm{FEV}_{1} \mathrm{AUC}_{0-3}$ responses (i.e., change from baseline) were greater in patients with GOLD 2 disease than GOLD 3-4 disease (Fig. 3a; Table S1 in the electronic supplementary material). In both of the GOLD subgroups, $\mathrm{FEV}_{1} \mathrm{AUC}_{0-3}$ responses were greater with both doses of tiotropium + olodaterol than the monotherapy components at Week 24, and differences were statistically significant for all comparisons analyzed (Table 3 ).

Trough $\mathrm{FEV}_{1}$ responses were consistently greater with both doses of tiotropium + olodaterol than the monotherapy components; no obvious trend was apparent between patients with GOLD 2 and GOLD 3-4 COPD (Fig. 4a). While all differences between the various monotherapies and tiotropium + olodaterol $5 / 5 \mu$ g were statistically significant in patients with GOLD 2 COPD, in GOLD 3-4 COPD patients receiving tiotropium + olodaterol $2.5 / 5 \mu \mathrm{g}$ the differences were not as robust, such that changes in trough $\mathrm{FEV}_{1}$ with tiotropium $5 \mu \mathrm{g}$ were not significantly different from tiotropium + olodaterol 2.5/5 $\mu \mathrm{g}$ (Table 3).

\section{Patients Receiving Prior LAMA or LABA}

\section{Maintenance Treatment}

In patients who received prior maintenance treatment, as in patients without prior LAMA or LABA maintenance bronchodilator therapy, the

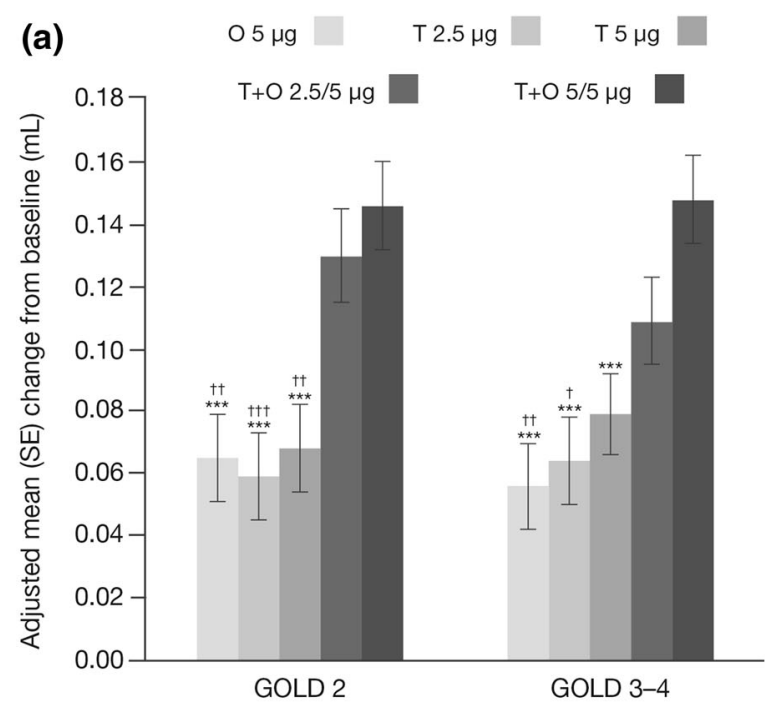

(b)

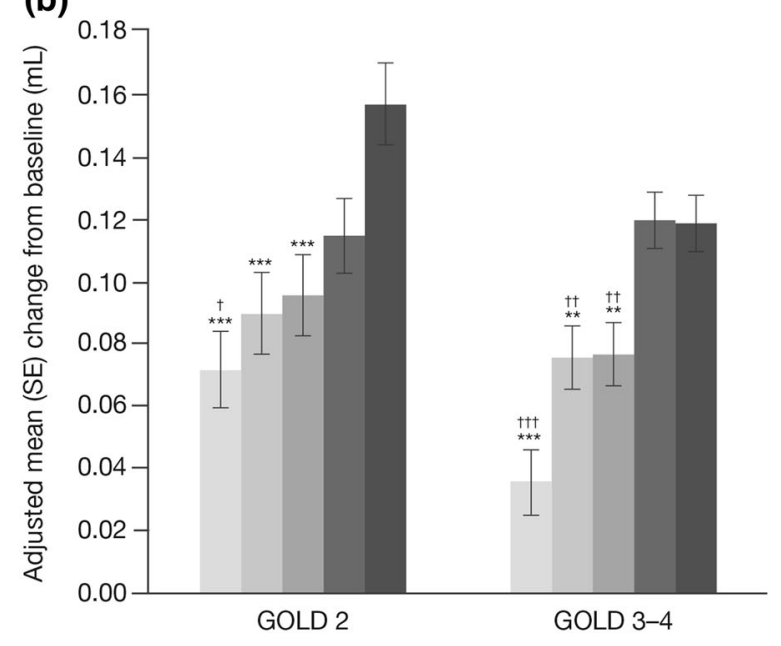

${ }^{* \star *} P<0.001$ versus $T+O 5 / 5 \mu \mathrm{g}$
${ }^{* \star} P<0.01$ versus $T+O 5 / 5 \mu \mathrm{g}$
${ }^{+t} P<0.001$ versus $T+O 2.5 / 5 \mu \mathrm{g}$
${ }^{+\dagger} P<0.01$ versus $T+O 2.5 / 5 \mu \mathrm{g}$
${ }^{+} P<0.05$ versus $T+O 2.5 / 5 \mu \mathrm{g}$

Fig. 4 Adjusted mean trough $\mathrm{FEV}_{1}$ responses at 24 weeks; pooled data from TONADO-1 and -2. a Patients without prior LAMA or LABA use; $\mathbf{b}$ patients with prior LAMA or LABA use. $F E V_{1}$ forced expiratory volume in $1 \mathrm{~s}$, $L A M A$ long-acting muscarinic antagonist, $L A B A$ longacting $\beta_{2}$-agonist, $S E$ standard error, $O$ olodaterol, $T$ tiotropium, GOLD Global initiative for chronic Obstructive Lung Disease

$\mathrm{FEV}_{1} \mathrm{AUC}_{0-3}$ responses for the individual treatments were greater in those with less severe COPD (Fig. 3b; Table S2 in the electronic 
supplementary material). Again, $\mathrm{FEV}_{1} \mathrm{AUC}_{0-3}$ responses were greater with both doses of tiotropium + olodaterol than the monotherapy components and all comparisons were statistically significant $(P<0.001)$.

Trough $\mathrm{FEV}_{1}$ responses were consistently greater with both doses of tiotropium + olodaterol than the monotherapy components and were generally greater in patients with less severe COPD (with the exception of tiotropium + olodaterol 2.5/5 $\mu$ g) (Fig. 4b). All comparisons between tiotropium + olodaterol and the monotherapies were statistically significant in patients with GOLD 3-4 COPD;

Table 4 Adjusted mean (SE) $\mathrm{FEV}_{1} \mathrm{AUC}_{0-3}$ and trough $\mathrm{FEV}_{1}$ responses (i.e., change from baseline) after 24 weeks of treatment (full analysis set): combined data for patients with prior LAMA or LABA treatment at baseline: treatment differences

\begin{tabular}{|c|c|c|c|c|c|c|}
\hline Treatment comparison & $\begin{array}{l}\text { Adjusted mean (SE) } \\
\mathrm{FEV}_{\mathbf{1}} \mathrm{AUC}_{0-3}, \mathrm{~mL}\end{array}$ & $95 \% \mathrm{CI}$ & $P$ value & $\begin{array}{l}\text { Adjusted mean (SE) } \\
\text { trough } \mathrm{FEV}_{1}, \mathrm{~mL}\end{array}$ & $95 \% \mathrm{CI}$ & $P$ value \\
\hline \multicolumn{7}{|l|}{ GOLD 2} \\
\hline \multicolumn{7}{|l|}{$\begin{array}{l}\text { Tiotropium + olodaterol } \\
5 / 5 \mu \mathrm{g}\end{array}$} \\
\hline versus olodaterol $5 \mu \mathrm{g}$ & $136(17)$ & 102,169 & $<0.0001$ & $85(18)$ & 50,121 & $<0.0001$ \\
\hline versus tiotropium $5 \mu \mathrm{g}$ & $123(17)$ & 89,157 & $<0.0001$ & $61(18)$ & 26,97 & 0.0007 \\
\hline \multicolumn{7}{|l|}{$\begin{array}{l}\text { Tiotropium + olodaterol } \\
2.5 / 5 \mu \mathrm{g}\end{array}$} \\
\hline versus olodaterol $5 \mu \mathrm{g}$ & $102(17)$ & 69,135 & $<0.0001$ & $43(17)$ & 9,77 & 0.0133 \\
\hline versus tiotropium $2.5 \mu \mathrm{g}$ & $113(17)$ & 81,146 & $<0.0001$ & $25(17)$ & $-9,59$ & 0.1567 \\
\hline versus tiotropium $5 \mu \mathrm{g}$ & $89(17)$ & 56,122 & $<0.0001$ & $19(18)$ & $-16,53$ & 0.2830 \\
\hline \multicolumn{7}{|l|}{ GOLD 3-4 } \\
\hline \multicolumn{7}{|l|}{$\begin{array}{l}\text { Tiotropium + olodaterol } \\
5 / 5 \mu \mathrm{g}\end{array}$} \\
\hline versus olodaterol $5 \mu \mathrm{g}$ & $119(14)$ & 92,146 & $<0.0001$ & $83(13)$ & 57,109 & $<0.0001$ \\
\hline versus tiotropium $5 \mu \mathrm{g}$ & $95(14)$ & 68,122 & $<0.0001$ & $41(14)$ & 15,68 & 0.0023 \\
\hline \multicolumn{7}{|l|}{$\begin{array}{l}\text { Tiotropium }+ \text { olodaterol } \\
2.5 / 5 \mu \mathrm{g}\end{array}$} \\
\hline versus olodaterol $5 \mu \mathrm{g}$ & $134(14)$ & 107,162 & $<0.0001$ & $85(14)$ & 58,111 & $<0.0001$ \\
\hline versus tiotropium $2.5 \mu \mathrm{g}$ & $107(14)$ & 80,134 & $<0.0001$ & $45(14)$ & 18,71 & 0.0010 \\
\hline versus tiotropium $5 \mu \mathrm{g}$ & $110(14)$ & 83,138 & $<0.0001$ & $43(14)$ & 16,70 & 0.0016 \\
\hline
\end{tabular}

Adjusted mean (SE) obtained from fitting a mixed model for repeated measurements including fixed effects of treatment, planned test day, treatment-by-test-day interaction, baseline, and baseline-by-test-day interaction; patient as a random effect; spatial power covariance structure for within-patient errors and Kenward-Roger approximation of denominator degrees of freedom

$S E$ standard error, $F E V_{1}$ forced expiratory volume in $1 \mathrm{~s}, A U C_{0-3}$ area under the curve from 0-3 h, LAMA long-acting muscarinic antagonist, $L A B A$ long-acting $\beta_{2}$-agonist, $C I$ confidence interval, GOLD Global initiative for chronic Obstructive Lung Disease 
(a)

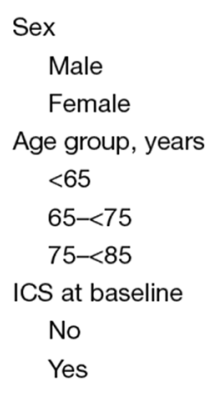

(b)

Sex

Male

Female

Age group, years

$<65$

$65-<75$

$75-<85$

ICS at baseline

No

Yes
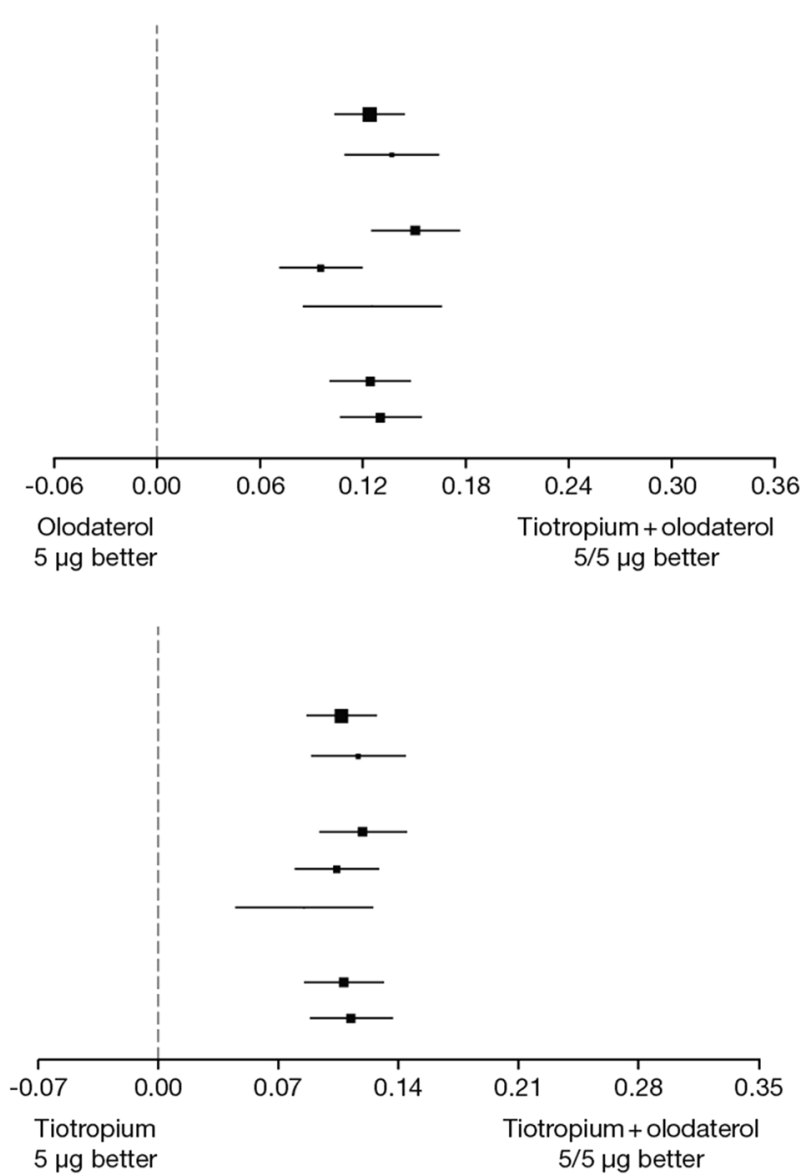

Fig. 5 Forest plots for $\mathrm{FEV}_{1} \mathrm{AUC}_{0-3}$ responses at 24 weeks. a Tiotropium + olodaterol $5 / 5 \mu \mathrm{g}$ versus olodaterol $5 \mu \mathrm{g}$; b tiotropium + olodaterol $5 / 5 \mu \mathrm{g}$ versus

however, the comparisons between tiotropium + olodaterol $\quad 2.5 / 5 \mu \mathrm{g}$ and tiotropium 2.5 or $5 \mu \mathrm{g}$ in patients with GOLD 2 COPD did not achieve statistical significance (Table 4).

\section{Other Patient Subgroups}

$\mathrm{FEV}_{1} \mathrm{AUC}_{0-3}$ and trough $\mathrm{FEV}_{1}$ responses at 24 weeks are presented by age, sex, and prior ICS usage in Figs. 5 and 6. These show that tiotropium + olodaterol was more effective than the monotherapy components across all of the subgroups analyzed, with the exception of trough $\mathrm{FEV}_{1}$ (tiotropium + olodaterol 5/5 $\mu \mathrm{g}$ tiotropium $5 \mu \mathrm{g} . F E V_{1}$ forced expiratory volume in $1 \mathrm{~s}$, $A U C_{0-3}$ area under the curve from 0 to $3 \mathrm{~h}, I C S$ inhaled corticosteroid

versus tiotropium $5 \mu \mathrm{g}$ ) in patients aged 75-85 years.

\section{DISCUSSION}

In all the patient subgroups included in this analysis of pooled data from the TONADO-1 and -2 studies, tiotropium + olodaterol consistently resulted in greater improvements in lung function than the monotherapy components, as determined by $\mathrm{FEV}_{1} \mathrm{AUC}_{0-3}$ and trough $\mathrm{FEV}_{1}$ responses at 24 weeks. These results are in line with data from other studies using a LAMA and a LABA, and further 

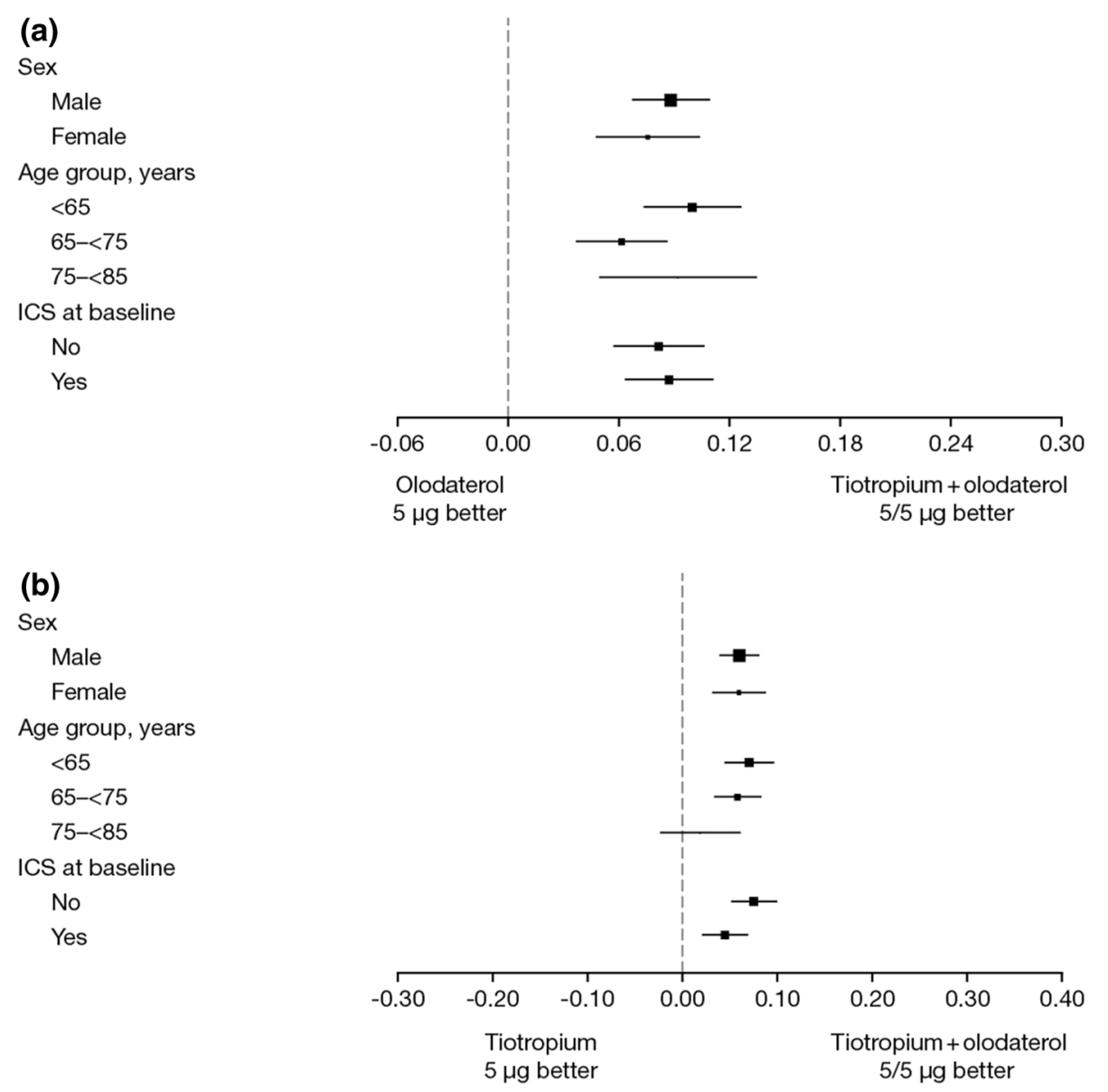

Fig. 6 Forest plots for trough $\mathrm{FEV}_{1}$ responses at 24 weeks. a Tiotropium + olodaterol $5 / 5 \mu \mathrm{g}$ versus olodaterol $5 \mu \mathrm{g}$; b tiotropium + olodaterol $5 / 5 \mu \mathrm{g}$ versus tiotropium $5 \mu \mathrm{g}$.

highlight the benefits of combined bronchodilation over monotherapy treatment [23-25]. Of particular note, tiotropium + olodaterol $5 / 5 \mu \mathrm{g}$ once daily improved lung function in patients with GOLD 2 and 3-4 disease, and there were no apparently relevant differences in lung-function responses according to whether these patients had or had not received prior maintenance therapy with a LAMA or a LABA.

While no statistical comparisons were made between subgroups, it was apparent that both $\mathrm{FEV}_{1} \mathrm{AUC}_{0-3}$ and trough $\mathrm{FEV}_{1}$ responses for the individual treatments were, in general, greater
$F E V_{1}$ forced expiratory volume in $1 \mathrm{~s}$, ICS inhaled corticosteroid

in patients with less severe disease. The greater lung-function responses observed in patients with GOLD 2 COPD in our studies are in line with other clinical trials, including the 4-year study of tiotropium (UPLIFT; NCT00144339) [18]. While patients are less symptomatic earlier in their disease course, recent data have suggested that the progression of early COPD occurs at a faster rate than during the later stages of disease [16, 17]. In addition, the UPLIFT study suggested a possible reduction in the rate of decline of post-bronchodilator $\mathrm{FEV}_{1}$ in patients with GOLD 2 COPD using tiotropium [18]. As more information becomes 
available related to the response of symptoms, lung function, and exacerbations to good maintenance bronchodilator therapy in COPD patients with less severe disease, opinion is moving towards using effective bronchodilation in the earlier stages of COPD $[18,26]$.

Our data support the use of dual-action maintenance bronchodilator therapy with LAMA + LABA for treatment-naive and less severe COPD, as well as those more severe patients already receiving maintenance therapies. Both doses of tiotropium + olodaterol were more effective than the monotherapy components in all subgroups analyzed, although the lower dose of the combination $(2.5 / 5 \mu \mathrm{g})$ may have been slightly less effective in providing trough bronchodilation in less severe, treatment-naive patients. In addition, it appeared that the $\mathrm{FEV}_{1}$ $\mathrm{AUC}_{0-3}$ responses were somewhat greater in younger patients than older patients, a finding that may reflect more the greater response in the less severe patients rather than a primary age effect. Otherwise, no major differences in responses were observed between treatment groups.

Our analysis does have some limitations in so far as it was post hoc and no direct statistical comparisons could be made between subgroups. However, based on the fact that data were pooled from two large phase III studies, the subgroups were all sizeable (each comprising $>900$ patients), allowing a clear evaluation of the effects of tiotropium + olodaterol in comparison to the monotherapy components.

\section{CONCLUSIONS}

Tiotropium + olodaterol $5 / 5 \mu \mathrm{g}$ resulted in improved lung-function responses compared to the monotherapy components across all subgroups analyzed. These data show that tiotropium + olodaterol is effective in patients irrespective of age, disease severity, and prior treatment with bronchodilators and/or ICS.

The improvements from baseline in lung function were greater in patients with less severe disease, adding support for the use of combination bronchodilation earlier in the course of COPD.

\section{ACKNOWLEDGMENTS}

The authors received no compensation related to the development of the manuscript. This work, article processing charges and open access fee were supported by Boehringer Ingelheim Pharma $\mathrm{GmbH} \&$ Co. KG. Medical writing assistance was provided by Rob Kite, on behalf of Complete HealthVizion, and was contracted and compensated by Boehringer Ingelheim Pharma GmbH \& Co. KG. All named authors meet the International Committee of Medical Journal Editors (ICMJE) criteria for authorship for this manuscript. They take full responsibility for the scope, direction, content of, and editorial decisions relating to, the manuscript, were involved at all stages of development, and have approved the submitted manuscript.

Conflict of interest. G.T. Ferguson has received grants, personal fees, and nonfinancial support from Boehringer Ingelheim and Forest, personal fees and non-financial support from GlaxoSmithKline, grants and personal fees from Novartis, AstraZeneca, and Pearl Therapeutics, and personal fees from Sunovion.

M. Fležar has received personal fees from Boehringer Ingelheim and institutional financial support from Novartis. 
S. Korn has received grants and personal fees from AstraZeneca, Boehringer Ingelheim, GlaxoSmithKline, Novartis, and Takeda, grants from Teva and MedImmune, and personal fees from Almirall and Grifols.

L. Korducki is an employee of Boehringer Ingelheim Pharmaceuticals Inc., Ridgefield, Connecticut, USA.

L. Grönke is an employee of Boehringer Ingelheim Pharma GmbH and Co. KG.

R. Abrahams has received grants and personal fees from Boehringer Ingelheim and GlaxoSmithKline, and grants from Forest, Pearl Therapeutics, and Pfizer.

R. Buhl has received grants and personal fees from Boehringer Ingelheim, Novartis, and Roche, and personal fees from AstraZeneca, Chiesei, Grifols, GlaxoSmithKline, and Takeda.

Compliance with ethics guidelines. Both studies were performed in accordance with the Declaration of Helsinki, International Conference on Harmonisation Harmonised Tripartite Guideline for Good Clinical Practice, and local regulations. The protocols were approved by the authorities and the ethics committees of the respective institutions, and signed informed consent was obtained from all patients.

This article does not contain any new studies with human or animal subjects performed by any of the authors.

Open Access. This article is distributed under the terms of the Creative Commons Attribution Noncommercial License which permits any noncommercial use, distribution, and reproduction in any medium, provided the original author(s) and the source are credited.

\section{REFERENCES}

1. Global Initiative for Chronic Obstructive Lung Disease. Global strategy for the diagnosis, management, and prevention of chronic obstructive pulmonary disease. Updated 2015. http://www.goldcopd.org/uploads/users/files/GO LD_Report_2015_Feb18.pdf. Accessed Mar 23, 2015.

2. Tashkin DP, Celli B, Senn S, et al. A 4-year trial of tiotropium in chronic obstructive pulmonary disease. N Engl J Med. 2008;359:1543-54.

3. Bateman ED, Tashkin D, Siafakas N, et al. A oneyear trial of tiotropium Respimat $^{\circledR}$ plus usual therapy in COPD patients. Respir Med. 2010;104:1460-72.

4. Cooper CB, Celli BR, Jardim JR, et al. Treadmill endurance during 2-year treatment with tiotropium in patients with COPD: a randomized trial. Chest. 2013;144:490-7.

5. Yohannes AM, Willgoss TG, Vestbo J. Tiotropium for treatment of stable COPD: a meta-analysis of clinically relevant outcomes. Respir Care. 2011;56:477-87.

6. Vogelmeier C, Hederer B, Glaab T, et al. Tiotropium versus salmeterol for the prevention of exacerbations of COPD. $\mathrm{N}$ Engl J Med. 2011;364:1093-103.

7. Wise RA, Anzueto A, Cotton D, et al. Tiotropium Respimat inhaler and the risk of death in COPD. N Engl J Med. 2013;369:1491-501.

8. Lange P, Aumann J-L, Derom E, et al. The 24-h FEV time profile of olodaterol QD delivered via Respimat $^{\circledR}$ in COPD: results from two 6-week studies. Eur Respir J. 2013;42 (Suppl 57):982s, abs 4635.

9. Feldman G, Bernstein JA, Hamilton A, Nivens C, LaForce C. The 24-hour $\mathrm{FEV}_{1}$ time profile of olodaterol once daily (QD) via Respimat ${ }^{\circledR}$ and formoterol twice daily (BID) via Aerolizer ${ }^{\circledR}$ in patients with COPD: results from two 6-week studies. Chest. 2013;144 (No. 4 Meeting Abstracts), abs 749A.

10. Ferguson G, Feldman G, Hofbauer P, et al. Lung function efficacy of olodaterol QD delivered via Respimat $^{\circledR}$ in COPD patients: results from two 48-week studies. Eur Respir J. 2013;42 (Suppl 57):5s, abs 187. 
11. Koch A, Pizzichini E, Hamilton A, et al. Lung function efficacy of olodaterol QD delivered via Respimat ${ }^{\circledR}$ vs placebo and formoterol BID in patients with COPD: two 48-week studies. Eur Respir J. 2013;42 (Suppl 57):146s, abs P764.

12. Koch A, Paggiaro P, Hamilton A, et al. Symptomatic benefit of olodaterol QD delivered via Respimat ${ }^{\circledR}$ vs placebo and formoterol BID in patients with COPD: combined analysis from two 48-week studies. Eur Respir J. 2013;42 (Suppl 57):145s, abs P763.

13. Maltais F, Kirsten A-M, Hamilton A, De Sousa D, Wang F, Decramer M. Evaluation of the effects of olodaterol on exercise endurance in patients with COPD: results from two 6-week studies. Chest. 2013;144 (No. 4 Meeting Abstracts), abs 748A.

14. Buhl R, Maltais F, Abrahams R, et al. Tiotropium and olodaterol fixed-dose combination versus mono-components in COPD (GOLD 2-4). Eur Respir J. 2015;45:969-79.

15. Derom E, Westerman J, Groenke L, Hamilton A, Li C, Beeh KM. The 24-hour lung function profile of once-daily tiotropium and olodaterol fixed-dose combination compared with placebo and monotherapies in chronic obstructive pulmonary disease. Am J Respir Crit Care Med. 2014;189, abs A6727.

16. Bridevaux P-O, Gerbase MW, Probst-Hensch NM, Schindler C, Gaspoz J-M, Rochat T. Long-term decline in lung function, utilisation of care and quality of life in modified GOLD stage 1 COPD. Thorax. 2008;63:768-74.

17. Vestbo J, Edwards LD, Scanlon PD, et al. Changes in forced expiratory volume in 1 second over time in COPD. N Engl J Med. 2011;365:1184-92.

18. Decramer M, Celli B, Kesten S, et al. Effect of tiotropium on outcomes in patients with moderate chronic obstructive pulmonary disease (UPLIFT): a prespecified subgroup analysis of a randomised controlled trial. Lancet. 2009;374:1171-8.
19. Maltais F, Dennis N, Chan CK. Rationale for earlier treatment in COPD: a systematic review of published literature in mild-to-moderate COPD. COPD. 2013;10:79-103.

20. Welte T, Vogelmeier C, Papi A. COPD: early diagnosis and treatment to slow disease progression. Int J Clin Pract. 2015;69:336-49.

21. Dahl R, Jadayel D, Alagappan VKT, Chen H, Banerji D. Efficacy and safety of QVA149 compared to the concurrent administration of its monocomponents indacaterol and glycopyrronium: the BEACON study. Int $\mathrm{J}$ Chron Obstruct Pulmon Dis. 2013;8:501-8.

22. Beeh K-M, Korn S, Beier J, et al. Effect of QVA149 on lung volumes and exercise tolerance in COPD patients: the BRIGHT study. Respir Med. 2014;108:584-92.

23. Mahler DA, D’Urzo A, Bateman ED, et al. Concurrent use of indacaterol plus tiotropium in patients with COPD provides superior bronchodilation compared with tiotropium alone: a randomised, double-blind comparison. Thorax. 2012;67:781-8.

24. Tashkin DP, Varghese ST. Combined treatment with formoterol and tiotropium is more efficacious than treatment with tiotropium alone in patients with chronic obstructive pulmonary disease, regardless of smoking status, inhaled corticosteroid use, baseline severity, or gender. Pulm Pharmacol Ther. 2011;24:147-52.

25. Bateman ED, Ferguson GT, Barnes N, et al. Dual bronchodilation with QVA149 versus single bronchodilator therapy: the SHINE study. Eur Respir J. 2013;42:1484-94.

26. Decramer $\mathrm{M}$, Dahl R, Kornmann O, Korn S, Lawrence D, McBryan D. Effects of long-acting bronchodilators in COPD patients according to COPD severity and ICS use. Respir Med. 2013;107:223-32. 